\title{
Homozygosity mapping: One more tool in the clinical geneticist's toolbox
}

\author{
Fowzan S. Alkuraya, $M D^{1,2,3}$
}

\begin{abstract}
Consanguinity increases the coefficient of inbreeding, which increases the likelihood of presence of pathogenic mutations in a homoallelic state. Although this is known to have an adverse outcome by increasing the risk of autosomal recessive disorders, this very phenomenon has also made homozygosity mapping the most robust gene discovery strategy in the recent history of human genetics. However, homozygosity mapping can also serve as an extremely powerful tool in the clinical genetics setting as well. In particular, this method is highly suited in the setting of genetically heterogeneous conditions and inborn errors of metabolism that require sophisticated biochemical testing that may not be readily available. This article is meant to highlight the clinical utility of this strategy using illustrative clinical examples from the author's own clinical genetics practice. Genet Med 2010:12(4):236-239.
\end{abstract}

Key Words: runs of homozygosity, genetic heterogeneity, inborn errors of metabolism

Co onsanguinity (union between related individuals) is an ancient practice that is still common in multiple cultures. ${ }^{1}$ Coefficient of inbreeding (percentage of genome that is homozygous or identical by descent) of the progeny of consanguineous union ranges from $<0.4$ (third cousin parents) to $12.5 \%$ (double first cousin parents). Therefore, although the impact of increased coefficient of inbreeding on the frequency of autosomal recessive conditions is well established, ${ }^{2-7}$ this same phenomenon increases the probability of disease-causing mutations to reside in blocks of homozygosity. This has made it possible for researchers to identify disease-causing mutations by pursuing hypothesis-free genomewide search for blocks of homozygosity. This has been particularly helpful in the setting of very rare disorders that are much more likely to present in a homozygous (more precisely autozygous) state than combined heterozygous state. ${ }^{8}$ Our group has similarly used this phenomenon to identify novel disease-causing genes. ${ }^{9,10}$ Increasingly, we have also realized that consanguineous populations lend themselves to "shortcuts" that make the practice of clinical genetics easier in a number of ways, again in the setting of autosomal recessive disorders. Genetically heterogeneous conditions, for example, represent a major challenge in outbred populations where prioritization scheme for mutation analysis

From the ${ }^{1}$ Department of Genetics, King Faisal Specialist Hospital and Research Center, Riyadh, Saudi Arabia; ${ }^{2}$ Department of Pediatrics, King Khalid University Hospital and College of Medicine, King Saud University, Riyadh, Saudi Arabia; and ${ }^{3}$ Department of Anatomy and Cell Biology, College of Medicine, Alfaisal University, Riyadh, Saudi Arabia.

Fowzan S. Alkuraya, MD, Developmental Genetics Unit, Department of Genetics, King Faisal Specialist Hospital and Research Center, MBC 03, PO Box 3354, Riyadh 11211 Saudi Arabia. E-mail: falkuraya@kfshrc.edu.sa.

Disclosure: The author declares no conflict of interest

Submitted for publication September 24, 2009.

Accepted for publication December 7, 2009

Published online ahead of print February 3, 2010.

DOI: $10.1097 /$ GIM.0b013e3181ceb95d usually follows published frequencies of the involved genes. In the setting of consanguineous parents, however, a much more efficient prioritization scheme is homozygosity scan, which is becoming increasingly easier and cheaper to perform - thanks to the advent of single nucleotide polymorphism (SNP) chipbased genotyping platforms. This scan can easily eliminate genes and often identify one or very few genes for downstream mutation analysis. In our experience, this has significantly reduced cost, impressively boosted the yield of mutation analysis, and dramatically enhanced patient care. This approach has allowed us to revisit genes that have been excluded by other centers on the basis of lack of identifiable mutation, only to have us uncover deep intronic mutations that we could only pursue because those were the only genes highlighted by the homozygosity scan.

\section{MATERIALS AND METHODS}

\section{Human subjects}

For the purpose of this review, all patients (inpatients and ambulatory) seen by the author from September 2007 to September 2009 were tabulated $(n=397)$. Illustrative cases are selected based on the presence of either of the following: (1) genetically heterogeneous condition or (2) suspected metabolic disease that requires invasive testing for confirmation. Referral pattern is typical of that seen in standard clinical genetics programs and includes syndromic evaluation, cognitive delay, abnormal newborn metabolic screening or other evidence of metabolic derangement, presence of one or more birth defects, positive family history, and abnormal prenatal findings.

\section{Homozygosity mapping}

In selected cases, homozygosity mapping was performed using SNP-based array. Affymetrix $10 \mathrm{~K}$ array platform was used until January 2008 when it was replaced by the denser $250 \mathrm{~K}$ array from the same manufacturer (Affymetrix, Santa Clara, CA). The process is straightforward and involves the extraction of genomic DNA from whole blood followed by a series of reactions using the manufacturer's protocol. Briefly, DNA samples go through the steps of digestion, adaptor ligation, whole genome polymerase chain reaction amplification, cleaning, fragmentation, labeling, and hybridization onto the SNP array using the Affymetrix GeneChip ${ }^{\circledR}$ System, which consists of a hybridization oven, GeneChip ${ }^{\circledR}$ Fluidics Station that automates the staining and washing of the probe array, and GeneArray ${ }^{\mathrm{TM}}$ Scanner that generates a real-time image of each chip for subsequent computational analysis. It is important to highlight that the internal normalization of the signal intensity generated by the different probes provides the advantage of inferring copy number in addition to genotype calling. We have been using two programs for the analysis of homozygosity blocks: Copy Number Analyzer for GeneChip ${ }^{\circledR}$ arrays Version $3.0^{11}$ and Affymetrix ${ }^{\circledR}$ Genotyping Console ${ }^{\mathrm{TM}}$ (Affymetrix, Santa Clara, CA). Obviously, with only two possible genotypes per SNP (generically referred to as A or B), one cannot assume 
any stretch of homoallelic calls to represent true homozygosity runs; so, these programs use special algorithms to highlight blocks that pass their statistical cutoff, which can be adjusted by the end user, of a bona fide run of $\mathrm{A} / \mathrm{A}$ and/or $\mathrm{B} / \mathrm{B}$ calls. In our experience, using the default settings for these programs has been sufficient to accurately call runs of homozygosity in the overwhelming majority of cases. In addition, as mentioned above, these two programs can infer from the probe signal intensity the presence of deletions or duplications, thus, the results can also satisfy the requirement for molecular karyotyping.

\section{RESULTS}

\section{Homozygosity mapping can obviate the need for specialized biochemical testing in the setting of metabolic disorders}

1. A 10-year-old boy was referred for evaluation because of coarse facial features, mental retardation, and elevated urinary level of glycosaminoglycans. Lack of corneal cloudiness narrowed the differential diagnosis of mucopolysaccharidosis (MPS) to MPSII (Hunter) or MPSIII (Sanfilippo). Homozygosity mapping highlighted one of the four known loci for MPSIII, and subsequent sequencing detected the presence of a homozygous pathogenic mutation in $N A G L U$ (p.R297X).

2. A 5-year-old boy with a neurodegenerative disorder and a positive family history was referred for further evaluation. Electron microscopy failed to reveal any abnormality. Homozygosity mapping revealed the presence of a block of homozygosity that harbors the recently described lipofuscinosis gene MFSD8. A novel missense mutation was identified and established the diagnosis of neuronal ceroid lipofuscinosis. ${ }^{12}$

3. Parents of a deceased infant with suspected peroxisomal biogenesis defects were referred to us for genetic counseling. It was not possible to perform complementation analysis because the only DNA left was from the deceased child. However, homozygosity mapping allowed us to identify the causative PEX mutation (Al-Dirbashi et al., unpublished data)

\section{Homozygosity mapping can minimize the need for sequencing multiple genes in the setting of genetically heterogeneous conditions}

1. We have previously described our experience with seven families that were referred for evaluation of possible diagnosis of Bardet-Biedl syndrome and how homozygosity mapping made it possible to identify the underlying mutation by sequencing only one gene per family. ${ }^{13}$

2. We have also previously described our experience with retinitis pigmentosa and how homozygosity mapping allowed us to identify the underlying mutation, with a detection rate of 51 of 52 patients by sequencing only 2 (of more than 30 genes identified to date) genes on average per patient. ${ }^{14}$

3. The mother of a deceased child with epidermolysis bullosa of unknown type was referred for counseling; she was at the end of the first trimester of her second pregnancy. We were able to locate a tissue block from the deceased child, and subsequent homozygosity mapping using DNA extracted from that block highlighted $L A M B 3$, and a novel mutation (p.T904I) was identified. This child had later died at the age of 4 months from complications of junctional epidermolysis bullosa.

\section{Homozygosity mapping can uncover previously unsuspected mutations}

A 4-year-old girl with Bardet-Biedl syndrome was tested in a major DNA diagnostic laboratory before referral and had negative $B B S 1$ mutation analysis. Homozygosity mapping highlighted $B B S 1$ as the only known $B B S$ gene to reside within any of the blocks of homozygosity identified. As a result, cDNA was generated for this gene, and a novel deep intronic mutation was indeed identified as a result. ${ }^{13}$

\section{Homozygosity mapping in gene discovery}

The literature is replete with gene discoveries made possible by homozygosity mapping. In fact, this method accounts for the majority of human disease-gene identifications that followed the landmark article by Lander and Botstein ${ }^{15}$ on the statistical

Table 1 Comparison of cost and turnaround time between a traditional diagnostic approach and the homozygosity mapping approach in the workup of the metabolic patients mentioned in the text

\begin{tabular}{|c|c|c|}
\hline & Traditional diagnostic approach & Homozygosity mapping approach \\
\hline MPS & $\begin{array}{l}\text { Skin biopsy }+ \text { fibroblast preparation }+ \text { assay of } 5 \\
\text { enzymes }+ \text { sequencing of one gene }\end{array}$ & $\mathrm{SNP}$ array + sequencing of $N A G L U$ \\
\hline Cost (\$) & $50+371+1390+300=2111$ & $400+300=700$ \\
\hline Duration (mo) & 6 & $2-3$ \\
\hline NCL & $\begin{array}{l}\text { Conjunctival biopsy }+ \text { E/M examination }+ \text { assay } \\
\text { for TPP } 1 \text { and PPT } 1+\text { sequencing of at least } 4 \text { genes }\end{array}$ & $\mathrm{SNP}$ array + sequencing of $M F S D 8$ \\
\hline Cost $(\$)$ & $200+300+271+4500=5271$ & $400+1250=1650$ \\
\hline Duration (mo) & $12-15$ & 4 \\
\hline PBD & Sequencing of a panel of $P E X$ genes & $\mathrm{SNP}$ array + sequencing of one $P E X$ gene \\
\hline Cost $(\$)$ & 3950 & $400+450=950$ \\
\hline Duration (mo) & $4-6$ & 3 \\
\hline
\end{tabular}

Cost of sequencing is estimated based on the average offered price listed on Geneclinics.org.

MPS, mucopolysaccharidosis; NCL, neuronal ceroid lipofuscinosis 7; PBD, peroxisomal biogenesis disorder; E/M, electron microscopy. 


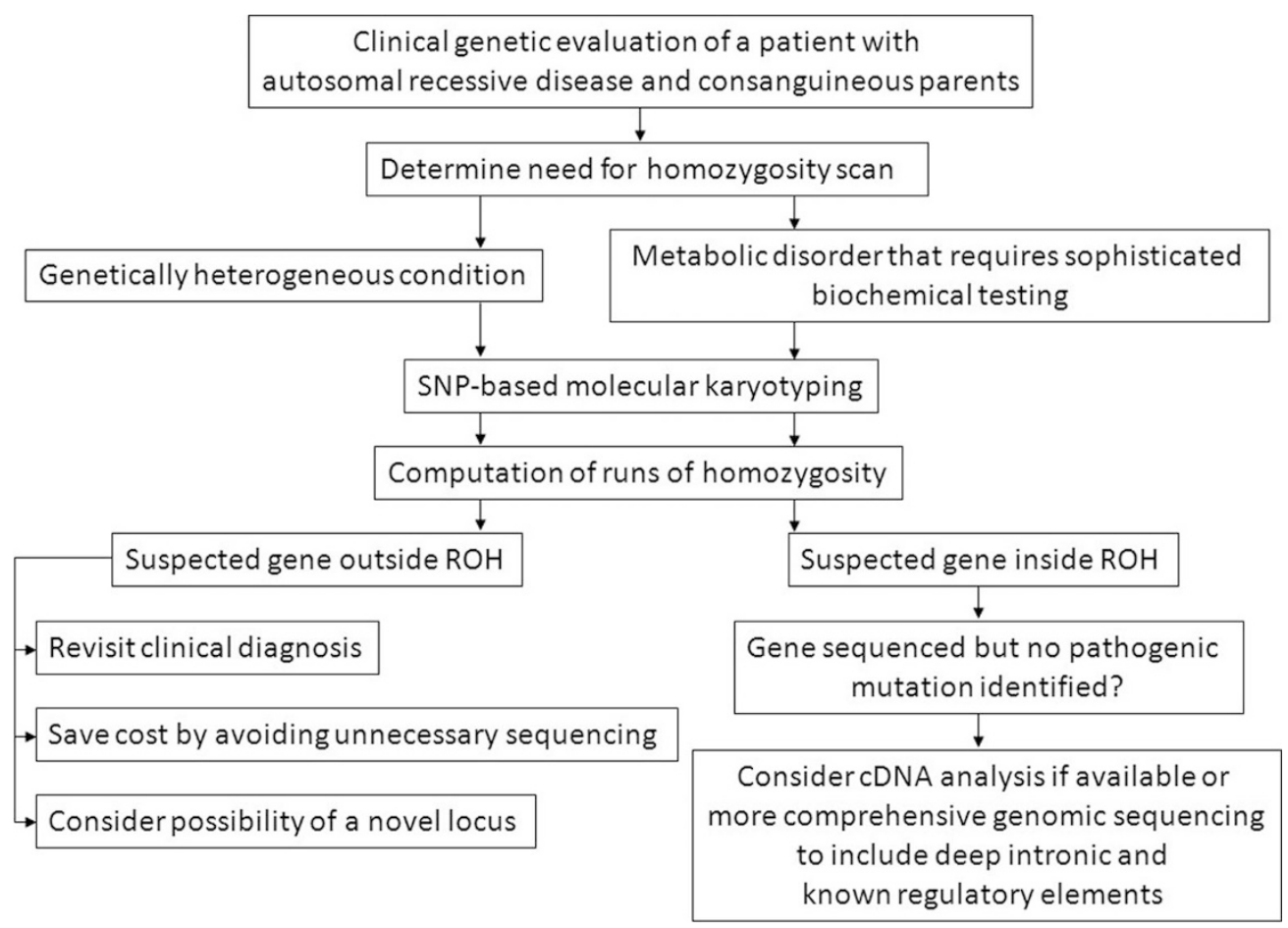

Fig. 1. Suggested algorithm for the use of homozygosity mapping in the practice of clinical genetics.

power provided by consanguineous families in the setting of linkage analysis. Because the scope of this article is to highlight the clinical rather than the research utility of homozygosity mapping, we will not provide further details on this application except to mention that even a single affected individual is theoretically sufficient to define a novel locus. This is particularly true in outbred populations where shared ancestors are likely to be many generations removed from the index case as elegantly shown recently by Hildebrandt et al. ${ }^{16}$

\section{DISCUSSION}

It should be clear from the above examples that homozygosity mapping has much more to offer to the clinical genetics community than its perceived role as a tool useful for research when multiple affected relatives are available. Indeed, as we have shown above, we have successfully used this technique to answer specific questions of great relevance to the daily practice of clinical genetics even when only one affected individual is available. The dramatic reduction in cost and turnaround time for identifying the underlying genetic defect is highlighted in Table 1, and a suggested algorithm for the use of this technique is shown in Figure 1. It must be stated that we do not suggest that our use of homozygosity mapping is novel. ${ }^{16-18}$ However, we do think that this technique is underused and believe several factors played a role in preventing the clinical genetics community from using it to its full potential. First, until recently, the only practical way to scan the genome for blocks of homozygosity was through the use of numerous microsatellite markers, an assay only available in specialized laboratories. Even when access to such laboratories is available, the tedious nature of this assay was not conducive to its routine clinical application. However, with the increasing use of SNP-based chip arrays for clinical cytogenetic analysis, we expect increasing interest among clinical geneticists in analyzing blocks of homozygosity as an extra "bonus" that comes with their order of molecular karyotyping. Second, clinical genetics as a discipline has historically evolved in countries where consanguinity was not common. Therefore, this technique may not have been viewed as applicable to the majority of patients. In our review of patients seen in our practice, consanguinity rate approached $90 \%$, well in excess of the national average of $56 \% .^{19}$ Thus, although consanguinity may not be common in many parts of the world, clinical geneticists should expect a bias toward overrepresentation of consanguineous couples in their practice. It is noteworthy that blocks of homozygosity that are of clinical relevance have recently been identified in populations that are not usually identified as consanguineous. ${ }^{20-22}$ These blocks represent distant shared ancestors and, as a consequence, are fewer and smaller in size, which makes it easier to pinpoint the culprit gene than in more consanguineous populations. ${ }^{16}$ Lastly, we believe that lack of sufficient "publicity" of the different utilities of homozygosity mapping and its frequent appearance in research articles that are concerned with disease gene identification have established a stereotype about this technique being of research utility only.

In summary, we have shown that consanguinity can be taken advantage of not only in the research arena but also, as importantly, in the practice of clinical genetics. We show that homozygosity scan is a valuable addition to the clinical geneticist's diagnostic toolbox. It is hoped that this article will spark interest among clinical geneticists to view this technique as such.

\section{ACKNOWLEDGMENTS}

I thank all members of my laboratory who made this work possible and for their support and hard work. 


\section{REFERENCES}

1. Jaber L, Halpern GJ, Shohat M. The impact of consanguinity worldwide. Community Genet 1998;1:12-17.

2. Becker SM, Al Halees Z, Molina C, Paterson RM. Consanguinity and congenital heart disease in Saudi Arabia. Am J Med Genet 2001;99:8-13.

3. Jaber L, Merlob P, Bu X, Rotter JI, Shohat M. Marked parental consanguinity as a cause for increased major malformations in an Israeli Arab community. Am J Med Genet 1992;44:1-6.

4. Rittler M, Liascovich R, Lopez-Camelo J, Castilla EE. Parental consanguinity in specific types of congenital anomalies. Am J Med Genet 2001;102:36-43.

5. Stoll C, Alembik Y, Dott B, Feingold J. Parental consanguinity as a cause of increased incidence of birth defects in a study of 131,760 consecutive births. Am J Med Genet 1994;49:114-117.

6. Stoltenberg C, Magnus P, Skrondal A, Lie RT. Consanguinity and recurrence risk of birth defects: a population-based study. Am J Med Genet 1999;82:423-428.

7. Zlotogora J. Genetic disorders among Palestinian Arabs: 1. Effects of consanguinity. Am J Med Genet 1997;68:472-475.

8. Aldahmesh MA, Abu-Safieh L, Khan AO, et al. Allelic heterogeneity in inbred populations: the Saudi experience with Alstrom syndrome as an illustrative example. Am J Med Genet A 2009;149A:662-665.

9. Alazami AM, Al-Saif A, Al-Semari A, et al. Mutations in C2orf37, encoding a nucleolar protein, cause hypogonadism, alopecia, diabetes mellitus, mental retardation, and extrapyramidal syndrome. Am J Hum Genet 2008;83:684-691.

10. Alazami AM, Shaheen R, Alzahrani F, et al. FREM1 mutations cause bifid nose, renal agenesis, and anorectal malformations syndrome. Am J Hum Genet 2009;85:414-418

11. Nannya Y, Sanada M, Nakazaki K, et al. A robust algorithm for copy number detection using high-density oligonucleotide single nucleotide polymorphism genotyping arrays. Cancer Res 2005;65:6071-6079.

12. Aldahmesh MA, Al-Hassnan ZN, Aldosari M, Alkuraya FS. Neuronal ceroid lipofuscinosis caused by MFSD8 mutations: a common theme emerging. Neurogenetics 2009;10:307-311.

13. Abu Safieh L, Aldahmesh M, Shamseldin $\mathrm{H}$, et al. Clinical and molecular characterization of Bardet-Biedl syndrome in consanguineous populations: the power of homozygosity mapping [published online ahead of print October 26, 2009]. J Med Genet. doi: 10.1136/jmg.2009.070755.

14. Aldahmesh MA, Safieh LA, Alkuraya H, et al. Molecular characterization of retinitis pigmentosa in Saudi Arabia. Mol Vis 2009;15:2464-2469.

15. Lander ES, Botstein D. Homozygosity mapping: a way to map human recessive traits with the DNA of inbred children. Science 1987;236:15671570 .

16. Hildebrandt F, Heeringa SF, Ruschendorf F, et al. A systematic approach to mapping recessive disease genes in individuals from outbred populations. PLoS Genet 2009;5:e1000353.

17. Megarbane A, Slim R, Nurnberg G, Ebermann I, Nurnberg P, Bolz HJ. A novel VPS13B mutation in two brothers with Cohen syndrome, cutis verticis gyrata and sensorineural deafness. Eur J Hum Genet 2009;17:1076-1079.

18. Littink KW, van Genderen MM, Collin RW, et al. A novel homozygous nonsense mutation in CABP4 causes congenital cone-rod synaptic disorder. Invest Ophthalmol Vis Sci 2009;50:2344-2350.

19. El Mouzan MI, Al Salloum AA, Al Herbish AS, Qurachi MM, Al Omar AA Consanguinity and major genetic disorders in Saudi children: a communitybased cross-sectional study. Ann Saudi Med 2008;28:169-173.

20. McQuillan R, Leutenegger AL, Abdel-Rahman R, et al. Runs of homozygosity in European populations. Am J Hum Genet 2008;83:359-372.

21. Nalls MA, Guerreiro RJ, Simon-Sanchez J, et al. Extended tracts of homozygosity identify novel candidate genes associated with late-onset Alzheimer's disease. Neurogenetics 2009;10:183-190.

22. Nalls MA, Simon-Sanchez J, Gibbs JR, et al. Measures of autozygosity in decline: globalization, urbanization, and its implications for medical genetics. PLoS Genet 2009;5:e1000415. 